\title{
HIGH-ENERGY EMISSION OF THE FIRST MILLISECOND PULSAR
}

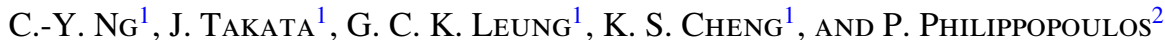 \\ ${ }^{1}$ Department of Physics, The University of Hong Kong, Pokfulam Road, Hong Kong, China; ncy@ bohr.physics.hku.hk \\ ${ }^{2}$ Department of Physics, McGill University, Montreal, QC H3A 2T8, Canada \\ Received 2014 March 8; accepted 2014 April 21; published 2014 May 15
}

\begin{abstract}
We report on X-ray and gamma-ray observations of the millisecond pulsar (MSP) B1937+21 taken with the Chandra $X$-ray Observatory, XMM-Newton, and the Fermi Large Area Telescope. The pulsar X-ray emission shows a purely non-thermal spectrum with a hard photon index of $0.9 \pm 0.1$, and is nearly $100 \%$ pulsed. We found no evidence of varying pulse profile with energy as previously claimed. We also analyzed $5.5 \mathrm{yr}$ of Fermi survey data and obtained much improved constraints on the pulsar's timing and spectral properties in gamma-rays. The pulsed spectrum is adequately fitted by a simple power-law with a photon index of $2.38 \pm 0.07$. Both the gamma-ray and X-ray pulse profiles show similar two-peak structure and generally align with the radio peaks. We found that the aligned profiles and the hard spectrum in X-rays seem to be common properties among MSPs with high magnetic fields at the light cylinder. We discuss a possible physical scenario that could give rise to these features.
\end{abstract}

Key words: gamma rays: stars - pulsars: general - pulsars: individual (PSR B1937+21 (J1939+2134)) - radiation mechanisms: non-thermal - X-rays: stars

Online-only material: color figures

\section{INTRODUCTION}

Millisecond pulsars (MSPs) are fast-spinning neutron stars with rotation periods $(P)$ from a few to tens of milliseconds. They are believed to be old pulsars spun up through an accretion process from a companion star. MSPs generally show small spin-down rates $(\dot{P})$ that imply low surface magnetic fields $\left(B_{s} \propto \sqrt{P \dot{P}}\right)$ of the order of $10^{8} \mathrm{G}$, much lower than those of young pulsars. Nonetheless, MSPs exhibit broadband emission as young pulsars do, and are detected across the electromagnetic spectrum from radio to X-ray to gamma-ray bands. Recently there has been significant progress in the highenergy studies of MSPs. The Large Area Telescope (LAT) on board the Fermi Gamma-ray Space Telescope has detected over 60 MSPs in gamma-rays since 2008 (see the LAT second pulsar catalog; Abdo et al. 2013). In addition, the Chandra X-ray Observatory and the XMM-Newton mission have provided sensitive measurements of the X-ray properties of over a dozen MSPs (see He et al. 2013, and references therein). These have significantly expanded the sample and allowed detailed studies of the population.

In this study we focus on the X-ray and gamma-ray emission properties of PSR B1937+21, which is a representative of an emerging class of MSPs that shows aligned pulse profiles in different energy bands (Guillemot et al. 2012). This could possibly indicate a different emission mechanism than that of typical pulsars. PSR B1937+21 (also known as PSR J1939+2134) is the first MSP discovered (Backer et al. 1982) and it remains the second fastest-spinning pulsar known, with $P=1.56 \mathrm{~ms}$ and $\dot{P}=1.05 \times 10^{-19}$. (Corrections of $\dot{P}$ due to proper motion and differential Galactic rotation are negligible.) These timing parameters suggest $B_{s}=4.1 \times 10^{8} \mathrm{G}$ and spin-down power $\dot{E}=4 \pi^{2} I \dot{P} / P^{3}=1.1 \times 10^{36} \mathrm{erg} \mathrm{s}^{-1}$, where $I=10^{45} \mathrm{~g} \mathrm{~cm}^{2}$ is neutron star moment of inertia. This is the second largest $\dot{E}$ among MSPs, only after PSR B1821-24.

At high energies, PSR B1937+21 was first detected in $\mathrm{X}$-rays with ASCA (Takahashi et al. 2001). The emission has a non-thermal spectrum and exhibits strong pulsations. The pulse profile shows a narrow peak structure with a pulsed fraction (PF) of $44 \%$. The study also suggests a hint of a second peak in the profile. Subsequent observations with RXTE and BeppoSAX confirmed the second peak (Cusumano et al. 2003; Nicastro et al. 2004), and it was claimed that the relative strength of the two peaks and the $\mathrm{PF}$ could vary with energy, from $\mathrm{PF}=85 \% \pm 5 \%$ in $1.3-10 \mathrm{keV}$ to $\mathrm{PF}=54 \% \pm 7 \%$ in $4-10 \mathrm{keV}$ (Nicastro et al. 2004). Zavlin (2007) reported on a Chandra observation of the source and found an X-ray photon index $\simeq 1.2$. Using $1.5 \mathrm{yr}$ of Fermi survey data, Guillemot et al. (2012) detected gammaray pulsations from PSR B1937+21. The pulse profile well aligns with the radio profile, indicating that both emission could originate from the same region in the outer magnetosphere. Spectral analysis using the Fermi data suggests that the phaseaveraged pulsar spectrum could be fitted with an exponentially cutoff power-law model (Guillemot et al. 2012). However, note that the spectral parameters of this source are not listed in the LAT second pulsar catalog that uses three years of data, due to low detection significance with a test-statistic (TS) value of 10 only (Abdo et al. 2013).

We present a new study of the high-energy emission of PSR B1937+21 using archival X-ray data made with Chandra and $X M M$ and $5.5 \mathrm{yr}$ of Fermi survey data. The observations and data reduction are described in Section 2 and the analysis and results are presented in Section 3. In Section 4, We compare the results with other MSPs and discuss possible physical emission mechanisms. Our findings are summarized in Section 5.

\section{OBSERVATIONS AND DATA REDUCTION}

We reprocessed the archival Chandra and XMM data. The former, which have been used in the previous study (Zavlin 2007), were taken on 2005 June 28 using the ACIS-S detector in the imaging mode and have a time resolution of $3.2 \mathrm{~s}$. The $X M M$ observation was taken on 2010 March 29 with the MOS1 and MOS2 cameras in the full frame mode that has a frame time of $2.6 \mathrm{~s}$ and the PN camera in the timing mode that has a high time resolution of $0.03 \mathrm{~ms}$. We performed the 
Table 1

Chandra and XMM Observations of PSR B1937+21

\begin{tabular}{|c|c|c|c|c|c|c|}
\hline Telescope & $\begin{array}{c}\text { Observation } \\
\text { Date }\end{array}$ & ObsID & Instrument & Mode & $\begin{array}{c}\text { Time Reso- } \\
\text { lution (s) }\end{array}$ & $\begin{array}{c}\text { Net Expo- } \\
\text { sure (ks) }\end{array}$ \\
\hline Chandra & 2005 Jun 28 & 5516 & ACIS-S & Faint & 3.2 & 49.5 \\
\hline \multirow[t]{3}{*}{$X M M$} & 2010 Mar 29 & 0605370101 & MOS1 & Full frame & 2.6 & 40.4 \\
\hline & & & MOS2 & Full frame & 2.6 & 47.5 \\
\hline & & & PN & Timing & $3 \times 10^{-5}$ & 40.4 \\
\hline
\end{tabular}

Chandra and XMM data reduction using CIAO 4.5 and SAS 12.0 , respectively. In the $X M M$ analysis, only PATTERN $\leqslant 12$ events from MOS and PATTERN $\leqslant 4$ events from PN were used to ensure good data quality. After removing periods of high background, we obtained net exposures of $49.5 \mathrm{ks}, 40.4 \mathrm{ks}$, $47.5 \mathrm{ks}$, and $40.4 \mathrm{ks}$ from Chandra, MOS1, MOS2, and PN, respectively. The observation parameters are listed in Table 1.

For the gamma-ray analysis, we selected Fermi LAT Pass 7 reprocessed data (P7REP; Bregeon et al. 2013) taken between 2008 August 4 and 2014 January 17. Class 2 events in the P7REP_SOURCE_V15 instrument response function (IRFs) were used throughout this paper, and the data reduction was carried out with the Fermi Science Tools v9r32p5. We restricted the analysis on data with zenith angles less than $100^{\circ}$, with spacecraft rocking angle less than $52^{\circ}$, and in the $0.1-100 \mathrm{GeV}$ energy range.

\section{ANALYSIS AND RESULTS}

\subsection{X-Ray Analysis}

PSR B1937+21 is clearly detected in both the Chandra and the $X M M$ observations, and the radial profiles of the images are consistent with a point source, same as what Zavlin (2007) found. Using a $3^{\prime \prime}$ radius aperture centered on the pulsar, we found $610 \pm 25$ background-subtracted Chandra counts from the in the $0.5-7 \mathrm{keV}$ energy range. Similarly, we extracted $370 \pm 20$ and $430 \pm 22$ net counts from MOS1 and MOS2, respectively, using a $20^{\prime \prime}$ radius aperture. The $\mathrm{PN}$ data have higher background since they were taken in the timing mode. We obtained $1230 \pm 70$ source counts from a $33^{\prime \prime}$ wide rectangular region in $0.5-7 \mathrm{keV}$.

\subsubsection{Timing}

We performed timing analysis with only the PN data, as they have a high time resolution. The photon arrival times were first corrected to the solar system barycenter, then folded using the TEMPO2 photons plug-in ${ }^{3}$ with the ephemeris from radio timing $^{4}$ (Smith et al. 2008). The resulting X-ray profile in the $0.5-7 \mathrm{keV}$ energy range is shown in Figure 1 . We have tried other energy bands between 0.5 and $10 \mathrm{keV}$, and found no energy dependence. The profile exhibits very sharp main pulse and interpulse components $\sim 180^{\circ}$ apart. In addition, there is a hint of a third peak in between, albeit it is not statistically significant. A direct comparison with the background level obtained from a nearby region indicates that the emission is nearly $100 \%$ pulsed. We also plotted the $1.4 \mathrm{GHz}$ radio profile in the figure for comparison. The X-ray pulse and interpulse slightly lag the radio ones.

To quantitatively measure the peak position and width, we followed Abdo et al. (2013) to fit the pulse profile using an

\footnotetext{
3 http://www.physics.mcgill.ca/ aarchiba/photons_plug.html

4 The timing parameter file is obtained from the LAT second pulsar catalog http://fermi.gsfc.nasa.gov/ssc/data/access/lat/2nd_PSR_catalog/.
}

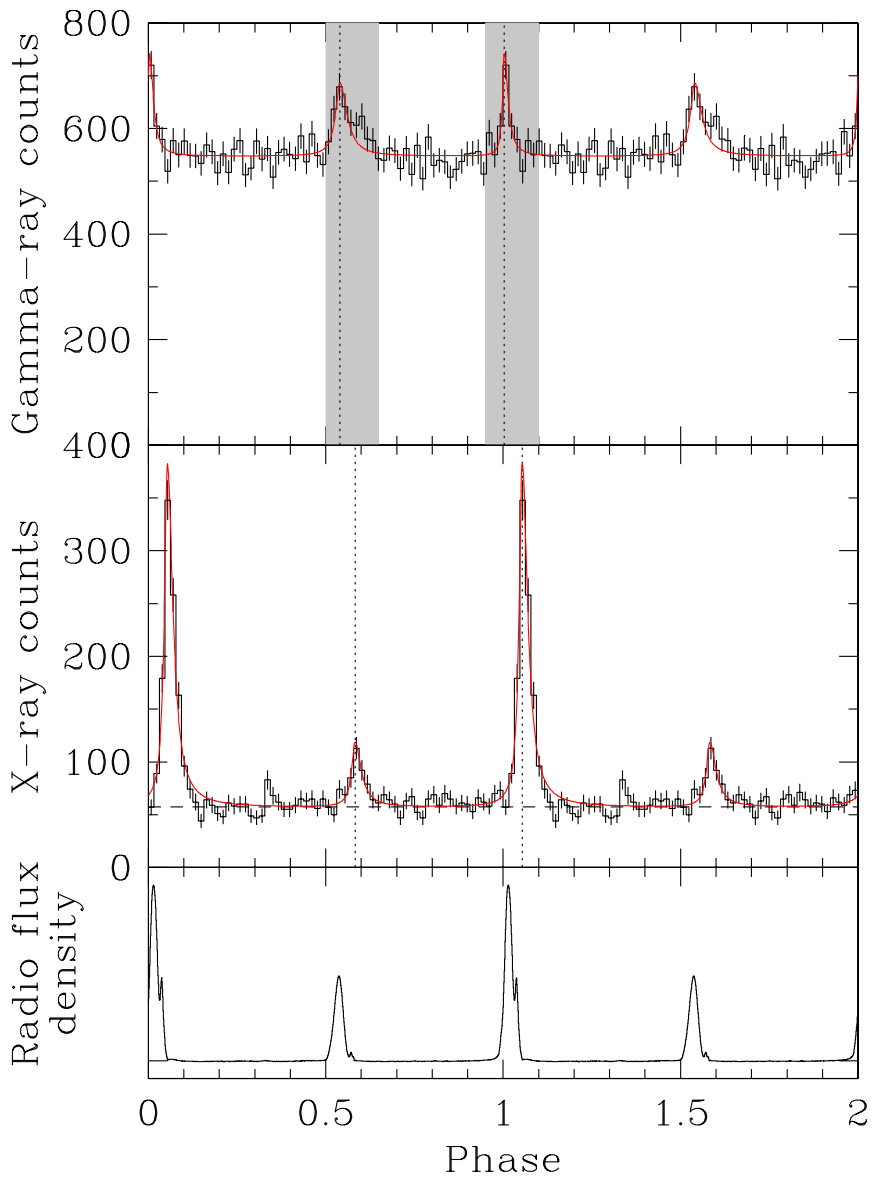

Figure 1. Pulse profiles of PSR B $1937+21$ in gamma-rays $(0.1-100 \mathrm{GeV}$; upper panel), X-rays (0.5-7 keV; middle panel), and radio $(1.4 \mathrm{GHz}$; lower panel). The latter is from Guillemot et al. (2012). The best-fit models with two asymmetric Lorentzians are shown by the red solid lines. The vertical dotted lines indicate the best-fit peak positions and the horizontal dashed line shows the X-ray background level. The shaded regions indicate the on-pulse phase intervals used for Fermi spectral analysis.

(A color version of this figure is available in the online journal.)

unbinned maximum likelihood method. We employed a simple model with two asymmetric Lorentzians. The functional form is given by

$$
g(x)=\frac{2}{\pi} \frac{A_{1}}{\left(\sigma_{1}^{-}+\sigma_{1}^{+}\right)\left(1+z_{1}^{2}\right)}+\frac{2}{\pi} \frac{A_{2}}{\left(\sigma_{2}^{-}+\sigma_{2}^{+}\right)\left(1+z_{2}^{2}\right)},
$$

where

$$
z_{i}=\left\{\begin{array}{ll}
\left(x-\Phi_{i}\right) / \sigma_{i}^{-} & \text {if } x \leqslant \Phi_{i} \\
\left(x-\Phi_{i}\right) / \sigma_{i}^{+} & \text {if } x>\Phi_{i}
\end{array},\right.
$$

$A_{i}$ are the amplitudes, $\Phi_{i}$ are the peak positions, and $\sigma_{i}^{ \pm}$are the width parameters such that the full widths at half-maximum (FWHMs) of the two peaks are given by $\sigma_{1}^{-}+\sigma_{1}^{+}$and $\sigma_{2}^{-}+\sigma_{2}^{+}$. 
Table 2

Best-fit Timing and Spectral Parameters of PSR B1937+21

\begin{tabular}{|c|c|c|}
\hline Parameter & X-ray & Gamma-ray \\
\hline \multicolumn{3}{|l|}{ Timing } \\
\hline \multicolumn{3}{|l|}{ First peak } \\
\hline Position, $\Phi_{1}$ & $0.054 \pm 0.001$ & $0.003_{-0.003}^{+0.001}$ \\
\hline Width parameter, $\sigma_{1}^{-}$ & $0.010_{-0.002}^{+0.001}$ & $0.006_{-0.004}^{+0.001}$ \\
\hline Width parameter, $\sigma_{1}^{+}$ & $0.019_{-0.003}^{+0.001}$ & $0.012_{-0.005}^{+0.001}$ \\
\hline $\mathrm{FWHM}_{1}=\sigma_{1}^{-}+\sigma_{1}^{+}$ & $0.029_{-0.003}^{+0.001}$ & $0.019_{-0.006}^{+0.002}$ \\
\hline Radio lag, $\delta_{1}$ & $0.068 \pm 0.001$ & $-0.011_{-0.004}^{+0.001}$ \\
\hline \multicolumn{3}{|l|}{ Second peak } \\
\hline Position, $\Phi_{2}$ & $0.583 \pm 0.005$ & $0.540_{-0.005}^{+0.006}$ \\
\hline Width parameter, $\sigma_{2}^{-}$ & $0.012_{-0.007}^{+0.003}$ & $0.015_{-0.007}^{+0.001}$ \\
\hline Width parameter, $\sigma_{2}^{+}$ & $0.020_{-0.009}^{+0.001}$ & $0.023_{-0.010}^{+0.001}$ \\
\hline $\mathrm{FWHM}_{2}=\sigma_{2}^{-}+\sigma_{2}^{+}$ & $0.032_{-0.012}^{+0.003}$ & $0.018_{-0.012}^{+0.002}$ \\
\hline Radio lag, $\delta_{2}$ & $0.032_{-0.005}^{+0.006}$ & $0.003_{-0.005}^{+0.006}$ \\
\hline \multicolumn{3}{|l|}{ Spectroscopy } \\
\hline Column density, $N_{\mathrm{H}}\left(10^{22} \mathrm{~cm}^{-2}\right)$ & $1.2 \pm 0.2$ & $\ldots$ \\
\hline Photon index, $\Gamma$ & $0.9 \pm 0.1$ & $2.38 \pm 0.07$ \\
\hline Statistic & $\chi_{v}^{2}=0.86$ & TS-value $=112$ \\
\hline Unabsorbed energy flux ${ }^{\mathrm{a}}\left(10^{-12} \mathrm{erg} \mathrm{cm}^{-2} \mathrm{~s}^{-1}\right)$ & $0.23_{-0.03}^{+0.04}$ & $16 \pm 2$ \\
\hline $\operatorname{Luminosity}^{\mathrm{a}}\left(10^{33} \mathrm{erg} \mathrm{s}^{-1}\right)$ & 0.7 & 49 \\
\hline Efficiency $^{\mathrm{a}}, \eta$ & $6 \times 10^{-4}$ & 0.044 \\
\hline
\end{tabular}

Notes. All uncertainties are statistical errors at the $1 \sigma$ confidence level. The timing parameters are in phase units between 0 and 1 .

${ }^{a}$ Phase-averaged values assuming no off-pulse emission. Also, no beaming correction has been applied. The X-ray and gamma-ray energy ranges are $0.5-7 \mathrm{keV}$ and $0.1-100 \mathrm{GeV}$, respectively.

We found that the main pulse and interpulse peak at phase $0.051 \pm 0.001$ and $0.583_{-0.005}^{+0.006}$, respectively. ${ }^{5}$ The best-fit results with statistical uncertainties at the $1 \sigma$ level are listed in Table 2 and the model profile is plotted in Figure 1. The uncertainties are estimated using Monte Carlo simulations. We generated 1000 random realizations of the best-fit model and fitted each one to obtain distributions of the best-fit parameters. The confidence intervals are determined from the most compact regions that contain $68 \%$ of the sample. Note that we did not account for any systematic uncertainties, such as the absolute timing accuracy of the instrument. Finally, we searched for bursts that may correspond to the giant pulses and any flux variabilities at longer timescale, but found negative result.

\subsubsection{Spectroscopy}

We extracted the pulsar spectrum from the regions stated above. Backgrounds were obtained from nearby regions on the same chip. As the pulsar is $100 \%$ pulsed, we extracted the PN spectrum from the on-pulse phase intervals only, between phase $0-0.15$ and $0.52-0.65$, in order to boost the signal-to-noise ratio. Note that there is a faint source CXO J193939.3+213506 located $13^{\prime \prime}$ northeast of the pulsar, which is not resolved by XMM. However, there should be negligible contamination to the pulsar spectrum, since its Chandra count rate in $0.5-7 \mathrm{keV}$ is only $2 \%$ that of the pulsar. This is also supported by the $\sim 100 \%$ PF of the PN counts.

The spectral fitting was performed in the Sherpa environment. We grouped the ACIS and MOS spectra to at least 20 counts per

\footnotetext{
5 Phase 0 is defined by the maximum of the first Fourier harmonic of the radio profile in the time domain (Guillemot et al. 2012).
}

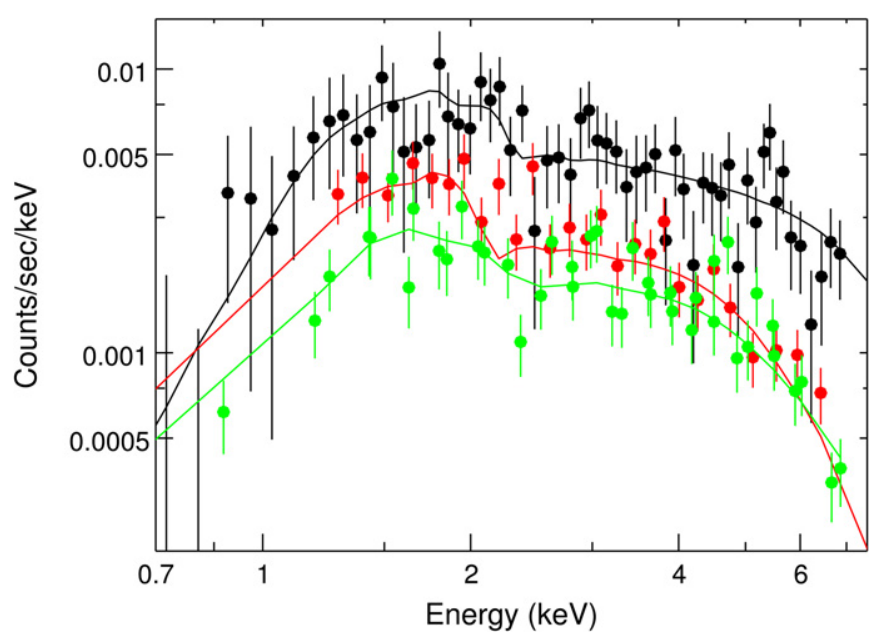

Figure 2. X-ray spectrum of PSR B1937+21. The Chandra, PN, and MOS1+MOS2 data are shown in red, black, and green, respectively. The solid lines indicate the best-fit absorbed power-law model.

(A color version of this figure is available in the online journal.)

bin, and the PN spectrum to at least 30 counts per bin. All four spectra (ACIS, MOS1, MOS2, and PN) were fitted jointly in the $0.5-7 \mathrm{keV}$ energy range. We employed a simple absorbed powerlaw model with abundances and the absorption cross sections given by Wilms et al. (2000). This provides a good fit with a reduced $\chi^{2}$ value of 0.86 over 122 degrees of freedom. The best-fit parameters are listed in Table 2 and the model is shown in Figure 2. The fit gives a column density of $N_{\mathrm{H}}=(1.2 \pm 0.2) \times$ $10^{22} \mathrm{~cm}^{-2}$ and a photon index of $\Gamma=0.9 \pm 0.1$ (all uncertainties are at the $1 \sigma$ confidence level). The absorbed and unabsorbed fluxes in $0.5-7 \mathrm{keV}$ are $(1.8 \pm 0.3) \times 10^{-13} \mathrm{erg} \mathrm{cm}^{-2} \mathrm{~s}^{-1}$ and $2.3_{-0.3}^{+0.4} \times 10^{-13} \mathrm{erg} \mathrm{cm}^{-2} \mathrm{~s}^{-1}$, respectively. For the source distance of $5 \mathrm{kpc}$ (Verbiest et al. 2012), this converts to X-ray luminosity of $L_{X}=6.8 \times 10^{32} \mathrm{erg} \mathrm{s}^{-1}$, implying an efficiency of $L_{X} / \dot{E}=6 \times 10^{-4}$. To check the cross-calibration, we fit the Chandra and $X M M$ data separately and obtained $N_{\mathrm{H}}=1.3_{-0.4}^{+0.5} \times 10^{22} \mathrm{~cm}^{-2}$ with $\Gamma_{X}=0.8 \pm 0.1$ from the former and $N_{\mathrm{H}}=(1.1 \pm 0.2) \times 10^{22} \mathrm{~cm}^{-2}$ with $\Gamma_{X}=1.0 \pm 0.2$ from the latter. These values are fully consistent.

We also tried a blackbody model, but the fit is slightly worse $\left(\chi_{v}^{2}=0.95\right)$ and the temperature seems too high $(k T=1.5 \mathrm{keV})$ to be physical. Moreover, adding a blackbody component to the power-law model shows no significant improvement to the fit. Nonetheless, we can still post a limit on the thermal emission by adjusting the blackbody parameters until the $\chi^{2}$ value exceeded a certain level. We obtained a temperature limit of $k T<0.13 \mathrm{keV}$ at the $99 \%$ confidence level, for any thermal emission from the polar cap region with a radius $r_{\mathrm{pc}}=R_{*} \sqrt{2 \pi R_{*} / c P}=3.7 \mathrm{~km}$, where $R_{*}=10 \mathrm{~km}$ is the neutron star radius.

\subsection{Gamma-Ray Analysis}

\subsubsection{Timing}

Using a $1^{\circ}$ radius region of interest (ROI) centered on the pulsar position, $3.7 \times 10^{4}$ events in the $0.1-100 \mathrm{GeV}$ energy range were extracted from the Fermi observation. We applied a barycentric correction to the photon arrival times and folded them using the TEMPO2 fermi plug-in, with the same radio ephemeris as for the X-ray analysis. Pulsations are clearly detected and the $H$-test (de Jager \& Büsching 2010) gives a statistic of 78 , which corresponds to $7.6 \sigma$ detection. The pulse 
profile is shown in Figure 1. We have also tried the $1-100 \mathrm{GeV}$ energy range and the profile looks very similar, albeit with a lower $H$ statistic of 19 only.

The gamma-ray profile in the figure shows sharp main pulse and interpulse that resemble the X-ray ones, but the gammaray pulses better align with the radio peaks. We fitted the gamma-ray profile with the same algorithm as described in the $\mathrm{X}$-ray analysis above and the best-fit parameters and statistical uncertainties are listed in Table 2. Finally, we checked the long term gamma-ray flux evolution of the source, and found no significant variability.

\subsubsection{Spectroscopy}

We performed a binned likelihood analysis using a $20^{\circ} \times 20^{\circ}$ square ROI. Our model includes all sources in the Second FermiLAT Source Catalog (2FGL; Nolan et al. 2012) within $20^{\circ}$ from the pulsar, the Galactic diffuse emission (gll_iem_v05.fits), and the extragalactic isotropic emission (iso_source_v05.txt). The source spectral models are all adopted from 2FGL. For sources located more than $8^{\circ}$ from the pulsar, their spectral parameters are held fixed during the fit. We have tried a power-law model and a power-law with exponential cutoff model (PLEC) for the pulsar spectrum. The latter has a functional form of

$$
\frac{d N}{d E} \propto E^{-\Gamma} \exp \left(-\frac{E}{E_{c}}\right),
$$

where $N$ is the photon flux and $E_{c}$ is the cutoff energy. We found that both models give low TS values that correspond to a detection significance below $3 \sigma$ level.

To boost the signal, we restricted the analysis on the onpulse data only, in phase $0.5-0.65$ and $0.95-1.1$. These intervals are indicated in Figure 1. We obtained a much higher TS value of 112 , i.e., above $10 \sigma$ significance, using the powerlaw model. The fit gives a photon index of $\Gamma_{\gamma}=2.38 \pm 0.07$. The best-fit parameters and the phase-averaged energy flux are listed in Table 2. We have also tried the PLEC model and found a slightly better fit (TS $=116$ ) with $\Gamma_{\gamma}=2.1 \pm 0.2$ and $E_{c}=8 \pm 4 \mathrm{GeV}$. However, the likelihood ratio test gives $-2 \Delta \log ($ likelihood $)=\Delta \mathrm{TS}<9$. We therefore follow the convention in Abdo et al. (2013) to conclude that the improvement is not significantly preferred. The best-fit powerlaw and PLEC models are plotted in Figure 3.

\section{DISCUSSION}

\subsection{Comparison with Previous Studies}

The Chandra and XMM observations offer better angular resolution and sensitivity than previous X-ray studies with $A S C A$, $R X T E$, and BeppoSAX, providing substantial improvements on the spectral and timing measurements. While $\Gamma_{X}$ we obtained is consistent with the reported values, we found a lower $N_{\mathrm{H}}$ of $1.2 \times 10^{22} \mathrm{~cm}^{-2}$ compared to $\sim 2 \times 10^{22} \mathrm{~cm}^{-2}$ given by Takahashi et al. (2001), Cusumano et al. (2003), and Nicastro et al. (2004). Our result is closer to the total Galactic H I column density of $1.1 \times 10^{22} \mathrm{~cm}^{-2}$ in the direction measured with radio observations (Kalberla et al. 2005). We however note that it is not unusual for sources near the Galactic plane to have an $\mathrm{X}$-ray absorption column density larger than the total $\mathrm{H}$ I column density, because X-rays are mostly absorbed by molecular clouds instead of neutral hydrogen atoms (see He et al. 2013). The small $N_{\mathrm{H}}$ value results in a slightly smaller unabsorbed

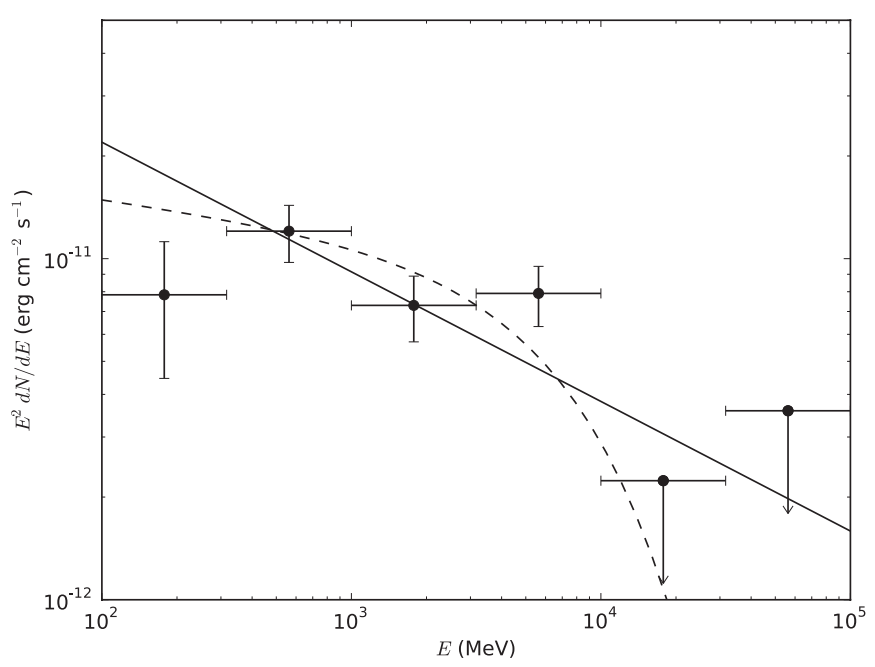

Figure 3. Pulsed Fermi gamma-ray spectrum of PSR B1937+21 obtained from binned maximum likelihood analysis. The best-fit power-law and power-law with exponential cutoff models are indicated by the solid and dashed lines, respectively. Statistical uncertainties at the $1 \sigma$ confidence level are shown here. The upper limits are at the $2 \sigma$ confidence level.

flux of $3.0 \times 10^{-13} \mathrm{erg} \mathrm{cm}^{-2} \mathrm{~s}^{-1}$ in $2-10 \mathrm{keV}$, as compared to $\sim 4 \times 10^{-13} \mathrm{erg} \mathrm{cm}^{-2} \mathrm{~s}^{-1}$ in Takahashi et al. (2001) and Nicastro et al. (2004).

For the timing analysis, the $X M M$ PN observation allows us to isolate the pulsar flux from the background emission, revealing for the first time the near $100 \% \mathrm{PF}$ of the X-ray emission. This is much higher than $\sim 60 \%$ found with $A S C A$ (Takahashi et al. 2001). Our results show no evidence of energydependent pulse profile in the soft X-ray band, thus rejecting the claim of a lower $(54 \% \pm 7 \%)$ PF below $4 \mathrm{keV}$ (Nicastro et al. 2004). The X-ray peak positions we obtained are mostly consistent with the published values. While a direct comparison with the RXTE results may seem to indicate slight offsets $\left(\Delta \phi_{1}=0.0028 \pm 0.001, \Delta \phi_{2}=0.0078 \pm 0.005\right)$, we note that these are smaller than the $40 \mu \mathrm{s}$ absolute timing accuracy of $X M M$ (Martin-Carrillo et al. 2012), which corresponds to 0.03 in phase units.

PSR B $1937+21$ is one of the few pulsars with radio giant pulses detected. Cusumano et al. (2003) noticed phase alignment between the radio giant and X-ray pulses, suggesting a possible correlation. Although there is no contemporaneous radio observation accompanied with the $X M M$ timing data, a large number of giant pulses are expected during the $11 \mathrm{hr}$ long X-ray exposure, given their observed rate of a few hundreds to a few thousands per hour (e.g., Soglasnov et al. 2004; Zhuravlev et al. 2013). We found no clustering of X-ray photon arrival times, same as what Cusumano et al. (2003) concluded. Similar negative results have been reported for other pulsars that show giant pulses (e.g., Johnston et al. 2004; Bilous et al. 2012).

Thanks to more Fermi data and the improved IRF (see Bregeon et al. 2013), our gamma-ray timing results are fully consistent with, but better constrained than, those from Guillemot et al. (2012). For the spectral analysis, we found a slightly harder photon index $\Gamma_{\gamma}=2.38 \pm 0.07$ compared to $3.02 \pm 0.18$ (Guillemot et al. 2012). On the other hand, our PLEC fit gives a softer $\Gamma_{\gamma}(2.1 \pm 0.2$ versus $1.43 \pm 0.87)$, although the two are formally compatible given the large uncertainties of the latter. We also obtained a higher cutoff energy at $E_{c}=8 \pm 4 \mathrm{GeV}$ as compared to $E_{c}=1.2 \pm 0.7 \mathrm{GeV}$. However, we note that $E_{c}$ is not very well determined in both cases. 
Table 3

High- $\dot{E}$ MSPs and Their X-ray Properties

\begin{tabular}{|c|c|c|c|c|c|c|c|c|c|}
\hline Name & $\begin{array}{c}P \\
(\mathrm{~ms})\end{array}$ & $\begin{array}{c}\dot{E} \\
\left(10^{35} \mathrm{erg} \mathrm{s}^{-1}\right)\end{array}$ & $\begin{array}{c}B_{s} \\
\left(10^{8} \mathrm{G}\right)\end{array}$ & $\begin{array}{c}B_{\mathrm{lc}} \\
\left(10^{5} \mathrm{G}\right)\end{array}$ & $\begin{array}{l}\text { Dist. } \\
(\mathrm{kpc})\end{array}$ & $\Gamma_{X}$ & $\begin{array}{c}L_{X} \\
\left(10^{32} \mathrm{erg} \mathrm{s}^{-1}\right)\end{array}$ & $\begin{array}{l}\mathrm{PF} \\
(\%)\end{array}$ & Ref. \\
\hline B1821-24 & 3.1 & 22 & 23 & 7.3 & 5.1 & $1.23 \pm 0.03$ & 14 & $82.5 \pm 4$ & 1 \\
\hline B1937+21 & 1.6 & 11 & 4.1 & 10 & 5.0 & $0.9 \pm 0.1$ & 6.8 & $\sim 100$ & This work \\
\hline B $1820-30 A$ & 5.4 & $8.3^{\mathrm{a}}$ & $43^{\mathrm{a}}$ & $2.5^{\mathrm{a}}$ & 7.9 & $\ldots$ & $\ldots$ & $\ldots$ & $\ldots$ \\
\hline $\mathrm{J} 1701-3006 \mathrm{~F}$ & 2.3 & $7.3^{\mathrm{a}}$ & $7.2^{\mathrm{a}}$ & $5.5^{\mathrm{a}}$ & 6.9 & $\ldots$ & $\ldots$ & $\cdots$ & $\cdots$ \\
\hline $\mathrm{J} 1701-3006 \mathrm{E}$ & 3.2 & $3.6^{\mathrm{a}}$ & $10^{\mathrm{a}}$ & $2.7^{\mathrm{a}}$ & 6.9 & $\ldots$ & $\cdots$ & $\cdots$ & $\cdots$ \\
\hline $\mathrm{J} 0218+4232$ & 2.3 & 2.4 & 4.3 & 3.1 & 2.7 & $1.10 \pm 0.06$ & 3.3 & $64 \pm 6$ & 2,3 \\
\hline B1957+20 & 1.6 & 1.1 & 1.4 & 3.0 & 2.5 & $\sim 2^{\mathrm{b}}$ & $\sim 0.5^{\mathrm{b}}$ & $\ldots$ & 4 \\
\hline $\mathrm{J} 1750-3703 \mathrm{D}$ & 5.1 & $1.4^{\mathrm{a}}$ & $16^{\mathrm{a}}$ & $1.1^{\mathrm{a}}$ & 12 & $<50$ & $\ldots$ & $\cdots$ & 5 \\
\hline B0021-72F & 2.6 & 1.4 & 4.1 & 2.1 & 4.0 & $\ldots$ & $0.055^{\mathrm{c}}$ & $\cdots$ & 6 \\
\hline $\mathrm{J} 1740-5340 \mathrm{~A}$ & 3.7 & $1.4^{\mathrm{a}}$ & $7.9^{\mathrm{a}}$ & $1.5^{\mathrm{a}}$ & 3.4 & $1.73 \pm 0.08^{b}$ & $0.22^{\mathrm{b}}$ & $\cdots$ & 7 \\
\hline $\mathrm{J} 1701-3006 \mathrm{D}$ & 3.4 & $1.2^{\mathrm{a}}$ & $6.6^{\mathrm{a}}$ & $1.5^{\mathrm{a}}$ & 6.9 & $\ldots$ & $\ldots$ & $\ldots$ & $\ldots$ \\
\hline
\end{tabular}

Notes.

a Observed value - there is no correction to $\dot{P}$ for the pulsar proper motion or differential Galactic rotation.

b The X-ray emission could be dominated by intra-binary shock (see the references).

c The X-ray spectrum is best fitted with a thermal model.

References. (1) Bogdanov et al. 2011; (2) Webb et al. 2004; (3) Kuiper et al. 2002; (4) Huang et al. 2012; (5) Becker et al. 2010; (6) Bogdanov et al. 2006; (7) Bogdanov et al. 2010.

\subsection{High- $\dot{E}$, High-B $B_{l c} M S P s$}

The non-thermal spectrum and large PF of the X-ray emission of PSR B1937+21 indicate a magnetospheric origin. These characteristics seem common among high- $\dot{E}$ MSPs (see Zavlin 2007). We list in Table 3 all known MSPs with $\dot{E}>10^{35} \mathrm{erg} \mathrm{s}^{-1}$. Three of them, PSRs B1821-24, B1937+21, and J0218+4232, have confirmed X-ray pulsations. ${ }^{6}$ They all exhibit hard powerlaw spectrum in X-rays with $\Gamma_{X} \sim 1$, large PF of $64 \%-100 \%$, general alignment between the X-ray and radio pulses (Kuiper et al. 2002; Johnson et al. 2013), and giant radio pulses (Romani \& Johnston 2001; Wolszczan et al. 1984; Knight et al. 2006). It has been suggested that these properties could be attributed to a strong magnetic fields at the light cylinder. Its strength, $B_{\mathrm{lc}}$, is related to the surface dipole field $B_{s}$ by $B_{\mathrm{lc}}=B_{s}\left(R_{*} / R_{\mathrm{lc}}\right)^{3}$, where $R_{\mathrm{lc}}=P c / 2 \pi$ is the light cylinder radius. All MSPs listed in the table have strong $B_{\mathrm{lc}}$ above $10^{5} \mathrm{G}$. In particular, PSR B1937+21 has the highest $B_{\text {lc }}$ of $1.0 \times 10^{6} \mathrm{G}$, even higher than that of the Crab pulsar.

In Table 4 we list all gamma-ray MSPs detected with Fermi LAT in decreasing order of $B_{\mathrm{lc}}$. We classified them according to their phase alignment between the gamma-ray and radio pulses, using a scheme similar to Venter et al. (2012): sources with gamma-ray peak lagging, aligned with, and preceding the radio peak are divided into classes I, II, and III, respectively. Class II pulsars are required to have a radio peak aligned with the gamma-ray peak to better than $1 / 10$ of the spin period, and the two peaks should have a similar profile. There are a small number of pulsars, e.g., PSR J2214+3000, that cannot be unambiguously classified. These cases are noted in the table. Finally, we also list in the table the X-ray spectral type of the MSPs, although only a few of them are detected since MSPs are generally faint. It is obvious that most high- $B_{\text {lc }}$ MSPs belong to class II and they exhibit non-thermal X-ray emission with hard spectra.

\footnotetext{
6 Guillemot et al. (2012) reported evidence of X-ray pulsations from PSR B $1957+20$, but the overall X-ray emission could be dominated by intra-binary shock emission (Huang et al. 2012).
}

\subsection{Modeling the Emission of High-B $B_{l c}$ MSPs}

Guillemot et al. (2012) first noted that a group of MSPs show aligned radio, X-ray, and gamma-ray pulse profiles, suggesting the same location for the emission. The authors proposed that the radio emission could be generated in caustics in the outer magnetosphere, same as the gamma-ray emission. As Table 4 indicates, the phase alignment is typical among the highest$B_{\mathrm{lc}}$ MSPs. We discuss below possible emission mechanisms in different energy bands, with a focus on their connection with $B_{\text {lc }}$. We qualitatively compare our toy model with the spectral energy distributions (SEDs) of three MSPs-PSRs B1937+21, B1821-24, and J0218+4232, which have been detected in both $\mathrm{X}$-rays and gamma-rays.

\subsubsection{Gamma-ray Emission}

We briefly summarize the gamma-ray emission mechanism in the context of the outer gap model (Cheng et al. 1986), in which electrons and positrons are accelerated up to a Lorentz factor of $\gamma \sim 10^{7}$ near the light cylinder. For a more detailed calculation of the gamma-ray emission process, we refer to Wang et al. (2010, 2011) and Takata et al. (2012). In the outer gap, electrons and positrons are accelerated by the electric field along the magnetic field lines and emit $\mathrm{GeV}$ gamma-rays via the curvature radiation process. The magnitude of the electric field is given by

$$
E_{\|} \sim \frac{f_{\mathrm{gap}} B_{\mathrm{lc}} R_{\mathrm{lc}}}{R_{c}}
$$

where $R_{c}$ is the curvature radius and $f_{\text {gap }}$ is the ratio between the gap thickness and the light cylinder radius, typically $f_{\text {gap }} \sim 0.3$ for MSPs (Takata et al. 2012).

The Lorentz factor of the accelerated particles can be estimated by the balance between the electric force and the back reaction force of the curvature radiation,

$$
\begin{aligned}
\gamma_{p}= & \left(\frac{3 R_{c}^{2}}{2 e} E_{\|}\right)^{1 / 4} \sim 5 \times 10^{6}\left(\frac{f_{\mathrm{gap}}}{0.3}\right)^{1 / 2}\left(\frac{P}{1 \mathrm{~ms}}\right)^{1 / 2} \\
& \times\left(\frac{B_{\mathrm{lc}}}{10^{5} \mathrm{G}}\right)^{1 / 4}\left(\frac{R_{c}}{R_{\mathrm{lc}}}\right)^{1 / 4} .
\end{aligned}
$$


Table 4

Properties of Fermi-LAT-detected MSPs

\begin{tabular}{|c|c|c|c|c|c|c|}
\hline Name & $\begin{array}{c}P \\
(\mathrm{~ms})\end{array}$ & $\begin{array}{c}\dot{E} \\
\left(10^{34} \mathrm{erg} \mathrm{s}^{-1}\right)\end{array}$ & $\begin{array}{c}B_{s} \\
\left(10^{8} \mathrm{G}\right)\end{array}$ & $\begin{array}{c}B_{\mathrm{lc}} \\
\left(10^{5} \mathrm{G}\right)\end{array}$ & $\begin{array}{c}\text { X-ray } \\
\text { Spectrum }^{a}\end{array}$ & $\begin{array}{c}\text { Radio/Gamma- } \\
\text { Ray Class }^{\text {b }}\end{array}$ \\
\hline B $1937+21$ & 1.6 & 110 & 4.1 & 9.9 & PL & II \\
\hline B1821-24 & 3.1 & 220 & 23 & 7.3 & PL & II \\
\hline $\mathrm{J} 0218+4232$ & 2.3 & 24 & 4.3 & 3.2 & PL & II \\
\hline $\mathrm{J} 1747-4036$ & 1.7 & $12^{\mathrm{c}}$ & $1.5^{\mathrm{c}}$ & $3.1^{\mathrm{c}}$ & $\ldots$ & $\mathrm{I} / \mathrm{II}$ \\
\hline B1957+20 & 1.6 & 7.6 & 1.2 & 2.5 & $\ldots$ & II \\
\hline B1820-30A & 5.4 & $83^{c}$ & $43^{c}$ & $2.5^{\mathrm{c}}$ & $\ldots$ & II \\
\hline J1902-5105 & 1.7 & $6.7^{\mathrm{c}}$ & $1.3^{\mathrm{c}}$ & $2.2^{\mathrm{c}}$ & $\ldots$ & II \\
\hline $\mathrm{J} 1810+1744$ & 1.7 & $4.0^{\mathrm{c}}$ & $0.9^{\mathrm{c}}$ & $1.8^{\mathrm{c}}$ & $\ldots$ & II \\
\hline $\mathrm{J} 1125-5825$ & 3.1 & $8.1^{\mathrm{c}}$ & $4.4^{\mathrm{c}}$ & $1.4^{\mathrm{c}}$ & $\ldots$ & I \\
\hline $\mathrm{J} 1446-4701$ & 2.2 & $3.7^{\mathrm{c}}$ & $1.5^{\mathrm{c}}$ & $1.3^{\mathrm{c}}$ & $\ldots$ & I \\
\hline $\mathrm{J} 2215+5135$ & 2.6 & $5.2^{\mathrm{c}}$ & $2.5^{\mathrm{c}}$ & $1.3^{\mathrm{c}}$ & $\cdots$ & I \\
\hline $\mathrm{J} 2241-5236$ & 2.2 & $3.3^{\mathrm{c}}$ & $1.4^{\mathrm{c}}$ & $1.2^{\mathrm{c}}$ & $\ldots$ & I \\
\hline J1658-5324 & 2.4 & $3.0^{\mathrm{c}}$ & $1.7^{\mathrm{c}}$ & $1.1^{\mathrm{c}}$ & $\ldots$ & I \\
\hline J0034-0534 & 1.9 & 1.7 & 0.75 & 1.0 & $\ldots$ & II \\
\hline $\mathrm{J} 1124-3653$ & 2.4 & $1.6^{\mathrm{c}}$ & $1.2^{\mathrm{c}}$ & $0.78^{\mathrm{c}}$ & $\cdots$ & III \\
\hline J0614-3329 & 3.2 & $2.2^{\mathrm{c}}$ & $2.4^{\mathrm{c}}$ & $0.71^{\mathrm{c}}$ & $\ldots$ & III \\
\hline J2043+1711 & 2.4 & 1.3 & 1.0 & 0.70 & $\cdots$ & I \\
\hline J0102+4839 & 3.0 & $1.8^{\mathrm{c}}$ & $1.9^{\mathrm{c}}$ & $0.67^{\mathrm{c}}$ & $\ldots$ & III \\
\hline $\mathrm{J} 2214+3000$ & 3.1 & $1.9^{\mathrm{c}}$ & $2.2^{\mathrm{c}}$ & $0.66^{\mathrm{c}}$ & $\ldots$ & II/III \\
\hline $\mathrm{J} 1858-2216$ & 2.4 & $1.1^{\mathrm{c}}$ & $1.0^{\mathrm{c}}$ & $0.66^{\mathrm{c}}$ & $\ldots$ & III \\
\hline J0023+0923 & 3.1 & $1.5^{\mathrm{c}}$ & $1.8^{\mathrm{c}}$ & $0.60^{c}$ & $\cdots$ & I \\
\hline $\mathrm{J} 2017+0603$ & 2.9 & $1.3^{\mathrm{c}}$ & $1.6^{\mathrm{c}}$ & $0.59^{c}$ & $\ldots$ & I \\
\hline $\mathrm{J} 1741+1351$ & 3.8 & 2.2 & 3.3 & 0.58 & $\cdots$ & III \\
\hline $\mathrm{J} 0101-6422$ & 2.6 & 1.0 & 1.1 & 0.58 & $\cdots$ & I \\
\hline J0613-0200 & 3.1 & 1.2 & 1.7 & 0.53 & $\cdots$ & I \\
\hline $\mathrm{J} 1514-4946$ & 3.6 & $1.6^{\mathrm{c}}$ & $2.6^{\mathrm{c}}$ & $0.52^{\mathrm{c}}$ & $\ldots$ & I \\
\hline J0751+1807 & 3.5 & 0.72 & 1.7 & 0.36 & $\ldots$ & 1 \\
\hline $\mathrm{J} 0340+4130$ & 3.3 & $0.65^{\mathrm{c}}$ & $1.4^{\mathrm{c}}$ & $0.36^{\mathrm{c}}$ & $\cdots$ & II/III \\
\hline $\mathrm{J} 2047+1053$ & 4.3 & $1.1^{\mathrm{c}}$ & $3.0^{\mathrm{c}}$ & $0.35^{\mathrm{c}}$ & $\cdots$ & III \\
\hline $\mathrm{J} 1600-3053$ & 3.6 & 0.73 & 1.8 & 0.35 & $\ldots$ & I \\
\hline $\mathrm{J} 1614-2230$ & 3.2 & 0.38 & 1.0 & 0.29 & $\cdots$ & I \\
\hline $\mathrm{J} 1231-1411$ & 3.7 & 0.51 & 1.6 & 0.29 & $\ldots$ & I \\
\hline J2051-0827 & 4.5 & 0.54 & 2.4 & 0.24 & $\ldots$ & I \\
\hline $\mathrm{J} 1744-1134$ & 4.1 & 0.41 & 1.7 & 0.23 & $\cdots$ & III \\
\hline $\mathrm{J} 1713+0747$ & 4.6 & 0.34 & 2.0 & 0.19 & $\ldots$ & I \\
\hline J2124-3358 & 4.9 & 0.37 & 2.4 & 0.18 & BB & III \\
\hline $\mathrm{J} 0030+0451$ & 4.9 & 0.35 & 2.3 & 0.18 & $\mathrm{BB}+\mathrm{PL}$ & I \\
\hline $\mathrm{J} 2302+4442$ & 5.2 & $0.38^{c}$ & $2.7^{\mathrm{c}}$ & $0.18^{\mathrm{c}}$ & $\ldots$ & $\mathrm{I} / \mathrm{II}$ \\
\hline $\mathrm{J} 0437-4715$ & 5.8 & 0.29 & 2.9 & 0.14 & $\mathrm{BB}+\mathrm{PL}$ & I \\
\hline J0610-2100 & 3.9 & 0.08 & 0.69 & 0.11 & $\ldots$ & III \\
\hline J1024-0719 & 5.2 & 0.05 & 0.92 & 0.06 & $\ldots$ & I \\
\hline
\end{tabular}

Notes. The pulsar parameters are all from the LAT second pulsar catalog (Abdo et al. 2013), except those of PSR B1821-24, which are from Johnson et al. (2013).

a Power-law (PL) or blackbody (BB) models.

b Alignment between the gamma-ray and radio pulse peaks. Class I: the gamma-ray pulse lags the radio pulse; class II: the gamma-ray and radio pulses are in phase; class III: the gamma-ray pulse precedes the radio one. Both classes are listed if a pulsar cannot be classified unambiguously. c Observed value - there is no correction to $\dot{P}$ for the pulsar proper motion or differential Galactic rotation.

The energy of the curvature photons is of the order of $\mathrm{GeV}$,

$$
\begin{aligned}
E_{c}= & \frac{3 h c \gamma^{3}}{4 \pi R_{c}} \sim 0.8\left(\frac{f_{\text {gap }}}{0.3}\right)^{1 / 4}\left(\frac{P}{1 \mathrm{~ms}}\right)^{1 / 2} \\
& \times\left(\frac{B_{\mathrm{lc}}}{10^{5} \mathrm{G}}\right)^{3 / 4}\left(\frac{R_{c}}{R_{\mathrm{lc}}}\right)^{-1 / 4} \mathrm{GeV}
\end{aligned}
$$

and the gamma-ray luminosity from the outer gap is typically

$$
\begin{aligned}
L_{\gamma} \sim & f_{\text {gap }}^{3} \dot{E} \sim 1.2 \times 10^{32}\left(\frac{f_{\text {gap }}}{0.3}\right)^{3}\left(\frac{P}{1 \mathrm{~ms}}\right)^{2} \\
& \times\left(\frac{B_{\mathrm{lc}}}{10^{5} \mathrm{G}}\right)^{2} \mathrm{erg} \mathrm{s}^{-1} .
\end{aligned}
$$

\subsubsection{X-Ray Emission}

In the standard pulsar theory, non-thermal X-ray emission is attributed to synchrotron radiation from secondary pairs in the outer magnetosphere, which are generated via the pair-creation process between gamma-rays and the background X-rays (e.g., Takata \& Chang 2007). The secondary pairs have an initial Lorentz factor of $\gamma_{\max } \sim(1 \mathrm{GeV}) /\left(2 m_{e} c^{2}\right) \sim 10^{3}$, then quickly lose their energy via synchrotron radiation and eventually leave the light cylinder with $\gamma_{\min } \sim 1 / \sin \theta_{s} \sim 10$, where $\theta_{s}$ is the pitch angle. During this process, synchrotron cooling gives non-thermal X-rays with $\Gamma_{X} \sim 1.5$ between $E_{\min } \sim 2 \times$ $10^{-2} \sin \theta_{s}\left(B_{\mathrm{lc}} / 10^{5} \mathrm{G}\right) \mathrm{keV}$ to $E_{\max } \sim 5 \sin \theta_{s}\left(B_{\mathrm{lc}} / 10^{5} \mathrm{G}\right) \mathrm{keV}$. This is the general case for young pulsars; they have $\Gamma_{X}$ observed 
in the range of 1.5-2 (e.g., Kuiper et al. 2001; Harding et al. 2002).

For high- $B_{\mathrm{lc}}$ MSPs, although non-thermal X-rays can be produced near the light cylinder, the very hard photon indices $\left(\Gamma_{X} \sim 1\right)$ are difficult to explain. A hard spectrum is expected below $E_{\min }$, but this is outside the observation bands. Harding et al. (2005) proposed that the primary particles in MSPs could maintain large momenta and undergo cyclotron resonant absorption of radio emission to produce synchrotron X-rays with a very hard spectrum. However, this process is not efficient between particles with $\gamma \sim 10^{7}$ and radio waves of $0.1-1 \mathrm{GHz}$, unless the $B$-field is of the order of $10^{7}-10^{8} \mathrm{G}$. Such a condition can only occur near the neutron star surface.

One possible scenario to explain the non-thermal X-rays from the outer magnetosphere is inverse-Compton (IC) scattering between the primary particles and radio waves. As we discussed, the radio, X-ray, and gamma-ray emission regions of high- $B_{\mathrm{lc}}$ MSPs are likely co-located. Therefore, the radio waves emitted in the outer magnetosphere may possibly irradiate the outer gap region and be up-scattered by the ultra-relativistic particles. We can estimate the energy density of the radio waves in the magnetosphere from the flux density. Assuming a radio spectral index of $\alpha=2$, the typical energy density of $\sim 100 \mathrm{MHz}$ radio waves is $U_{\mathrm{ph}} \sim 10^{4}-10^{5}$ erg $\mathrm{cm}^{-3}$ in high- $B_{\mathrm{lc}}$ MSPs. The radiation power of IC scattering from a single particle is then $P_{\mathrm{IC}} \sim 4 \sigma_{T} c \gamma^{2} U_{\mathrm{ph}} / 3$, where $\sigma_{T}$ is the Thomson cross section. As a comparison, the power of the curvature radiation is $P_{\text {cur }} \sim 2 e^{2} c \gamma^{4} / 3 R_{c}^{2}$. Hence,

$$
\frac{P_{\mathrm{IC}}}{P_{\text {cur }}} \sim 0.1\left(\frac{U_{p h}}{10^{4} \mathrm{erg} \mathrm{cm}^{-3}}\right)\left(\frac{\gamma}{10^{7}}\right)^{-2}\left(\frac{R_{c}}{10^{7} \mathrm{~cm}}\right)^{2} .
$$

This suggests that the expected energy flux from the IC process is only slightly smaller than that of the $\mathrm{GeV}$ emission and it could be observable in high- $B_{\mathrm{lc}}$ MSPs.

To model the IC spectrum, we consider the scattering between outgoing particles in the outer gap and outwardly propagating radio waves. We assume that the radio emission region lies just above the outer gap and approximate the magnetic field lines by concentric circles. The collision angle between the primary particles and radio waves can be crudely estimated by $\sin \theta_{0} \sim \sqrt{2 f_{\text {gap }}}$. For each particle, the IC power per unit energy per unit solid angle is given by

$$
\frac{d P_{\mathrm{IC}}}{d \Omega} \sim \mathcal{D}^{2}\left(1-\beta \cos \theta_{0}\right) F_{\mathrm{rad}} \frac{d \sigma^{\prime}}{d \Omega^{\prime}},
$$

where $d \sigma^{\prime} / d \Omega^{\prime}$ is the differential Klein-Nishina cross section, $\beta=\sqrt{\gamma^{2}-1} / \gamma, \mathcal{D}=\gamma^{-1}\left(1-\beta \cos \theta_{1}\right)^{-1}, 0<\theta_{1}<1 / \gamma$ is the angle between the particle motion and the scattered photon direction, and $F_{\text {rad }}$ is the radio spectrum.

The IC spectrum depends sensitively on the radio spectrum. Although the latter is not very clear at low radio frequency $<1 \mathrm{GHz}$, it is believed that a spectral turnover should exist below $100 \mathrm{MHz}$ for MSPs (see Kuzmin \& Losovsky 2001), which is lower than that of young pulsars $(\sim 1 \mathrm{GHz}$; e.g., Kijak et al. 2007). For a turnover at $10-100 \mathrm{MHz}$, the corresponding break in the IC spectrum would be at $(1-10) \times\left(\gamma / 10^{7}\right)^{2} \mathrm{MeV}$. This is well above the Chandra and XMM energy bands, suggesting that the IC emission could contributed to the observed X-rays. To compare with observations, we assume a broken power-law spectrum in radio with a turnover at $10 \mathrm{MHz}$, i.e.,

$$
F_{\text {rad }}(v)=A \begin{cases}\left(\frac{v}{100 \mathrm{MHz}}\right)^{\beta_{1}} & \text { for } v \geqslant 10 \mathrm{MHz} \\ \left(\frac{10 \mathrm{MHz}}{100 \mathrm{MHz}}\right)^{\beta_{1}}\left(\frac{v}{10 \mathrm{MHz}}\right)^{\beta_{2}} & \text { for } v<10 \mathrm{MHz} .\end{cases}
$$

The spectral index $\beta_{1}$ is inferred from the observed flux densities at $400 \mathrm{MHz}$ and $1.4 \mathrm{GHz}$ listed in the ATNF pulsar catalog (Manchester et al. 2005). The index below $10 \mathrm{MHz}$, $\beta_{2}$, is taken to be 0.5 , which provides a good match to the $\mathrm{X}$-ray spectra (see below). The normalization $A$ is related to the observed flux density at $400 \mathrm{MHz}\left(F_{400}\right)$ and the source distance $d$ by

$$
A \sim F_{400}\left(\frac{d}{R_{\mathrm{lc}}}\right)^{2}\left(\frac{100 \mathrm{MHz}}{400 \mathrm{MHz}}\right)^{\beta_{1}} .
$$

As the exact geometry of the radio emission region is unknown, we allowed $A$ to vary by a factor of a few to fit the observed SEDs.

We also considered synchrotron X-rays contributed by the secondary pairs. The particles accelerated toward the star would eventually reach the stellar surface and heat up the polar cap region. Takata et al. (2012) estimated a surface temperature of $\sim 1 \mathrm{MK}$ and a luminosity of $L_{r} \sim 5 \times 10^{31} \mathrm{erg} \mathrm{s}^{-1}$ for the thermal emission of PSR B1937+21. These values are below our detection limit of $1.5 \mathrm{MK}$. The thermal X-rays may collide with the GeV gamma-rays to create new pairs, which emit nonthermal X-rays via the synchrotron process. The optical depth of the pair-creation process is $\tau_{X \gamma} \sim L_{r} \sigma_{X \gamma} R_{\mathrm{lc}} /\left(4 \pi R_{\mathrm{lc}}^{2} c k T_{r}\right) \sim$ 0.02 , where $k_{B}$ is the Boltzmann constant and $\sigma_{X \gamma} \sim \sigma_{T} / 3$. The energy distribution of the secondary pairs follows

$$
\frac{d N_{e}}{d \gamma_{s}}\left(\gamma_{s}\right) \sim \frac{m_{e} c^{2}}{\dot{E}_{\mathrm{syn}}} \int_{2 \gamma_{s} m_{e} c^{2}}^{\infty} Q\left(E_{\gamma}^{\prime}\right) d E_{\gamma}^{\prime},
$$

where $\gamma_{s}$ is the Lorentz factor of the secondary pairs, $Q\left(E_{\gamma}\right)=$ $F_{\text {cur }}\left(1-e^{-\tau_{X \gamma}}\right) / E_{\gamma}, F_{\text {cur }}$ is the curvature radiation power per unit energy, and $\dot{E}_{\text {syn }}$ is the rate of energy loss from synchrotron radiation. With $\dot{E}_{\text {syn }}=2 e^{4} B^{2} \sin ^{2} \theta_{s} \gamma_{s}^{2} / 3 m_{e}^{2} c^{3}$, Equation (12) describes a power-law with index $p=2$. The synchrotron spectrum from secondary pairs is

$$
F_{\text {syn }}\left(E_{\gamma}\right)=\frac{\sqrt{3} e^{3} B \sin \theta_{s}}{h m_{e} c^{2}} \int \frac{d N_{e}}{d \gamma_{s}} F(x) d \gamma_{s},
$$

where $x=E_{\gamma} / E_{\mathrm{syn}}, E_{\mathrm{syn}}=3 h e \gamma_{s}^{2} B \sin \theta_{s} / 4 \pi m_{e} c$, and $\sin \theta_{s} \sim \sqrt{2 f_{\text {gap }}}$.

In Figure 4 we compare our simple model with the observed SEDs of PSRs B1937+21, B1821-24, and J0218+4232. It shows that our model provides reasonable fits to the data. The peak flux of the IC and curvature radiation are similar and the IC emission dominates over the synchrotron radiation in the X-ray band above a few $\mathrm{keV}$. Since the IC spectrum is sensitive to the radio spectral index, low-frequency radio measurements below $100 \mathrm{MHz}$ in future can offer essential inputs to refine the modeling. For the assumed spectral turnover at $10 \mathrm{MHz}$, the IC emission peaks at $\sim 100 \mathrm{keV}$, which could be detectable with hard X-ray telescopes such as NuSTAR or ASTRO-H.

\subsubsection{Radio Emission}

The radio emission process in pulsars is not clearly understood, nonetheless, it has been suggested that plasma instability 

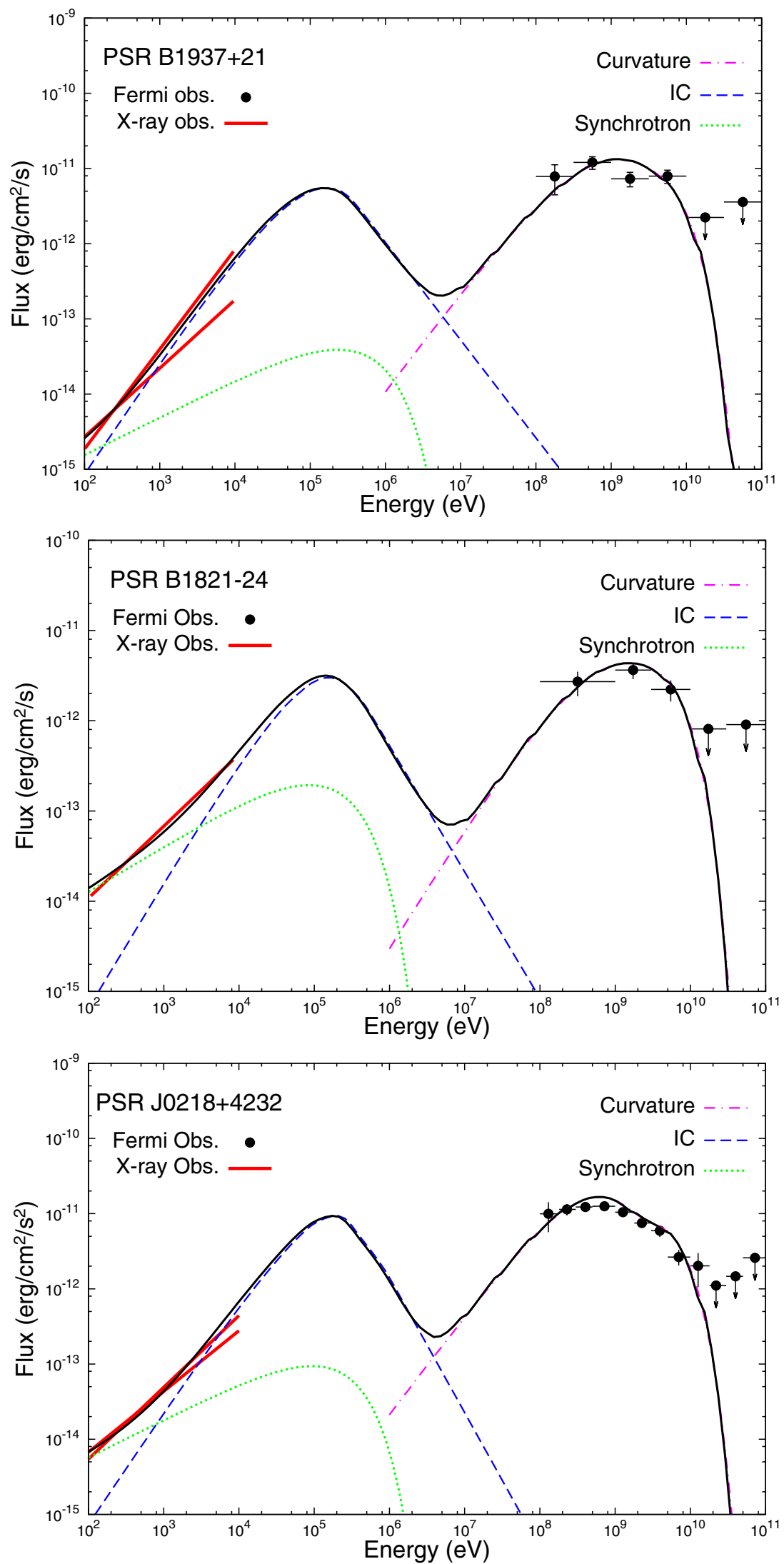

Figure 4. Our emission model compared with the observed SEDs of PSRs B1937+21, B1821-24, and J0218+4232 from X-rays to gamma-rays. The thick red line and the dots show the X-ray and gamma-ray data, respectively. The curvature radiation, IC emission, synchrotron emission, and total emission of the model are indicated by the magenta dashed-dotted lines, blue dashed lines, green dotted lines, and black lines, respectively.

(A color version of this figure is available in the online journal.) 
could play an important role in the generation of coherent radio emission (e.g., Usov 1987). The characteristic timescale for the instabilities (e.g., two-stream instability) to develop is related to the inverse of the plasma frequency $\omega_{p}=\sqrt{4 \pi e^{2} n_{e} / m_{e}}$. The electron and positron number density, $n_{e}$, is proportional to the Goldreich-Julian charge density $\left(n_{\mathrm{GJ}}\right)$ such that $n_{e} \propto n_{\mathrm{GJ}} \sim$ $B_{\mathrm{lc}} /(2 \pi P c)$. Therefore, high- $B_{\mathrm{lc}}$ MSPs tend to have a higher plasma frequency, and hence a shorter instability timescale, in the outer magnetosphere.

Above the outer gap accelerator, the outgoing and incoming gamma-rays produce electron-positron pairs moving toward the light cylinder and the star, respectively. These flows could develop two-stream instability in the outer magnetosphere. Since there are more pairs created near the inner boundary than near the outer boundary of the outer gap (Takata et al. 2004), the outflow is stronger than the inflow at the light cylinder. The Lorentz factor of the two flows are of the order of $\gamma_{s} \sim 10$, for which the synchrotron cooling timescale is comparable to light-crossing time of the magnetosphere. The instability development timescale can be estimated by

$$
\tau_{i} \sim\left(\frac{n_{o}}{n_{i}}\right)^{1 / 3} \gamma_{s}^{3 / 2} \omega_{p, o}^{-1}
$$

where $n_{o}$ and $n_{i}$ are the number densities of the outgoing and incoming flows, respectively, and $\omega_{p, o}$ is the plasma frequency of the outgoing flow (Usov 1987). With a typical multiplicity of the order of $\kappa \sim 10^{3}$, we may assume $n_{o} \sim \kappa n_{\mathrm{GJ}}$ and $n_{i} \sim n_{\mathrm{GJ}}$ in the outer magnetosphere. Hence, the timescale becomes

$$
\tau_{i} \sim 1.4 \times 10^{-8}\left(\frac{\kappa}{10^{3}}\right)^{-1 / 6}\left(\frac{P}{1 \mathrm{~ms}}\right)^{1 / 2}\left(\frac{B_{\mathrm{lc}}}{10^{5} \mathrm{G}}\right)^{-1 / 2} \mathrm{~s}
$$

which is much shorter than the light-crossing time of $\tau_{c} \sim$ $R_{\mathrm{lc}} / c \sim 1.6 \times 10^{-4}(P / 1 \mathrm{~ms}) \mathrm{s}$. As a result, the instability could develop before the outgoing particles escape the magnetosphere. We speculate that the two-stream instability may generate nonhomogeneous and separated plasma clouds, and the scattering or emission process of the plasma cloud eventually produce the observed radio emission in phase with the gamma-ray pulses.

Finally, we note that some high- $B_{\text {lc }}$ MSPs, e.g., PSRs B1821-24 and $\mathrm{J} 1810+1744$, show complex radio profiles with additional components that offset from the gamma-ray peaks. These could be contributed by emission from the polar cap region as in the conventional theory of pulsar radio emission.

\section{CONCLUSIONS}

We have analyzed X-ray and gamma-ray observations of PSR B1937+21 taken with Chandra, XMM, and Fermi LAT. We obtained much improved spectral and timing measurements than previous studies. Our results show that the pulsar $\mathrm{X}$-ray emission is $\sim 100 \%$ pulsed and has a purely non-thermal spectrum that can be described by a hard power law of photon index $\Gamma_{X}=0.9 \pm 0.1$. The X-ray pulse profile consists of two sharp peaks $\sim 180^{\circ}$ apart. They generally align with the radio peaks and the phase offsets are less than $7 \%$ of the spin period. In gamma-rays, the 5.5 years of Fermi survey data provide a good quality pulse profile in $0.1-100 \mathrm{GeV}$ with a significance of over $7 \sigma$. We performed a binned likelihood analysis on the pulsed emission and found that a simple power-law model with $\Gamma_{\gamma}=2.38 \pm 0.07$ gives a TS value of 112 , corresponding to over $10 \sigma$ significance. Adding an exponential cutoff to the power-law model slightly improves the fit, but the change is not statistically significant.

A comparison of PSR B1937+21 with other MSPs indicates that sources with a strong magnetic field at the light cylinder tend to show a hard, non-thermal X-ray spectrum and good alignment of pulse profiles in different energy bands. The latter suggests that the radio, $\mathrm{X}$-ray, and gamma-ray emission could originate from the same region in the outer magnetosphere. We speculate that radio emission could be generated in the outer gap region when $B_{\mathrm{lc}}$ is large, since this could give rise to short instability time scales. We investigate a simple model in which the nonthermal X-rays are contributed by IC scattering between radio waves and primary particles in the outer magnetosphere and by synchrotron radiation from secondary particles. We showed that this toy model is capable of qualitatively reproducing the observed SEDs of the highest- $B_{\text {lc }}$ MSPs. Future observations at low radio frequencies and in hard $\mathrm{X}$-rays can help refine the modeling.

We thank the referee for careful reading and useful suggestions and thank Vicky Kaspi and Anne Archibald for useful discussions. J.T., G.C.K.L., and K.S.C. are supported by a GRF grant of the Hong Kong Government under HKU7009/11P.

Facilities: $C X O$ (ACIS), XMM (EPIC), Fermi (LAT)

\section{REFERENCES}

Abdo, A. A., Ajello, M., Allafort, A., et al. 2013, ApJS, 208, 17

Backer, D. C., Kulkarni, S. R., Heiles, C., Davis, M. M., \& Goss, W. M. 1982, Natur, 300, 615

Becker, W., Huang, H. H., \& Prinz, T. 2010, arXiv:1006.0335

Bilous, A. V., McLaughlin, M. A., Kondratiev, V. I., \& Ransom, S. M. 2012, ApJ, 749,24

Bogdanov, S., Grindlay, J. E., Heinke, C. O., et al. 2006, ApJ, 646, 1104

Bogdanov, S., van den Berg, M., Heinke, C. O., et al. 2010, ApJ, 709, 241

Bogdanov, S., van den Berg, M., Servillat, M., et al. 2011, ApJ, 730, 81

Bregeon, J., Charles, E., Wood, et al. (for the Fermi-LAT Collaboration). 2013, in Proc. 4th Fermi Symposium, Monterey, California, 2012, eConf C121028, ed. T. J. Brandt, N. Omodei, \& C. Wilson-Hodge (arXiv:1304.5456)

Cheng, K. S., Ho, C., \& Ruderman, M. 1986, ApJ, 300, 500

Cusumano, G., Hermsen, W., Kramer, M., et al. 2003, A\&A, 410, L9 de Jager, O. C., \& Büsching, I. 2010, A\&A, 517, L9

Guillemot, L., Johnson, T. J., Venter, C., et al. 2012, ApJ, 744, 33

Harding, A. K., Strickman, M. S., Gwinn, C., et al. 2002, ApJ, 576, 376

Harding, A. K., Usov, V. V., \& Muslimov, A. G. 2005, ApJ, 622, 531

He, C., Ng, C.-Y., \& Kaspi, V. M. 2013, ApJ, 768, 64

Huang, R. H. H., Kong, A. K. H., Takata, J., et al. 2012, ApJ, 760, 92

Johnson, T. J., Guillemot, L., Kerr, M., et al. 2013, ApJ, 778, 106

Johnston, S., Romani, R. W., Marshall, F. E., \& Zhang, W. 2004, MNRAS, 355,31

Kalberla, P. M. W., Burton, W. B., Hartmann, D., et al. 2005, A\&A, 440, 775

Kijak, J., Gupta, Y., \& Krzeszowski, K. 2007, A\&A, 462, 699

Knight, H. S., Bailes, M., Manchester, R. N., Ord, S. M., \& Jacoby, B. A. 2006, ApJ, 640, 941

Kuiper, L., Hermsen, W., Cusumano, G., et al. 2001, A\&A, 378, 918

Kuiper, L., Hermsen, W., Verbunt, F., et al. 2002, ApJ, 577, 917

Kuzmin, A. D., \& Losovsky, B. Y. 2001, A\&A, 368, 230

Manchester, R. N., Hobbs, G. B., Teoh, A., \& Hobbs, M. 2005, AJ, 129, 1993

Martin-Carrillo, A., Kirsch, M. G. F., Caballero, I., et al. 2012, A\&A, 545, A126

Nicastro, L., Cusumano, G., Löhmer, O., et al. 2004, A\&A, 413, 1065

Nolan, P. L., Abdo, A. A., Ackermann, M., et al. 2012, ApJS, 199, 31

Romani, R. W., \& Johnston, S. 2001, ApJL, 557, L93

Smith, D. A., Guillemot, L., Camilo, F., et al. 2008, A\&A, 492, 923

Soglasnov, V. A., Popov, M. V., Bartel, N., et al. 2004, ApJ, 616, 439

Takahashi, M., Shibata, S., Torii, K., et al. 2001, ApJ, 554, 316

Takata, J., \& Chang, H.-K. 2007, ApJ, 670, 677

Takata, J., Cheng, K. S., \& Taam, R. E. 2012, ApJ, 745, 100

Takata, J., Shibata, S., \& Hirotani, K. 2004, MNRAS, 354, 1120

Usov, V. V. 1987, ApJ, 320, 333

Venter, C., Johnson, T. J., \& Harding, A. K. 2012, ApJ, 744, 34 
Verbiest, J. P. W., Weisberg, J. M., Chael, A. A., Lee, K. J., \& Lorimer, D. R. 2012, ApJ, 755, 39

Wang, Y., Takata, J., \& Cheng, K. S. 2010, ApJ, 720, 178

Wang, Y., Takata, J., \& Cheng, K. S. 2011, MNRAS, 414, 2664

Webb, N. A., Olive, J.-F., \& Barret, D. 2004, A\&A, 417, 181

Wilms, J., Allen, A., \& McCray, R. 2000, ApJ, 542, 914
Wolszczan, A., Cordes, J., \& Stinebring, D. 1984, in Birth and Evolution of Neutron Stars: Issues Raised by Millisecond Pulsars, ed. S. P. Reynolds \& D. R. Stinebring (Green Bank, WV: NRAO), 63

Zavlin, V. E. 2007, Ap\&SS, 308, 297

Zhuravlev, V. I., Popov, M. V., Soglasnov, V. A., et al. 2013, MNRAS, 430,2815 